Max Wuehr, PhD

Thomas Brandt, MD

Roman Schniepp, MD

Correspondence to

Dr. Wuehr:

Max.Wuehr@med.unimuenchen.de

\title{
Distracting attention in phobic postural vertigo normalizes leg muscle activity and balance
}

\section{ABSTRACT}

Objective: To examine the triggering causes of inadequate neuromuscular regulation of posture and subjective imbalance in patients with phobic postural vertigo (PPV), a subtype of functional dizziness.

Methods: Postural performance was assessed by center-of-pressure displacements and surface EMG of lower-limb muscles (the tibialis anterior and soleus) in 10 patients with PPV and 10 healthy controls under 4 stance conditions: standing with eyes open or closed and with or without an additional cognitive dual task. The level of muscle cocontraction and the characteristics of open- and closed-loop postural control were analyzed.

Results: At baseline (i.e., standing with eyes open without dual task), patients exhibited increased muscle cocontractions ( $p=0.003$ ), which were further associated with increased open-loop diffusion activity $(p=0.022)$ and a lowering of the primary feedback threshold for closed-loop control ( $p=0.003$ ). However, postural performance of patients improved considerably and normalized to that of healthy controls when performing an additional dual task.

Conclusions: PPV is characterized by a dissociation of subjective postural instability and objectively maintained balance capabilities. The dual-task effects on balance in patients with PPV indicate that this dissociation might result from an increased attention to postural adjustments at baseline, which is normally required only during demanding balance situations. This internal focus on balance control promotes an inappropriate neuromuscular regulation of posture, with increased muscle cocontractions, higher short-term body sway, and an oversensitivity to external stimuli. However, if patients are distracted, muscle cocontractions and balance control normalize. Such distraction may therefore be an effective coping strategy for preventing PPV attacks in susceptible patients. Neurology ${ }^{\circledR}$ 2017;88:284-288

\section{GLOSSARY}

HC = healthy control; PPV = phobic postural vertigo; $\mathbf{R M S}=$ root mean square; SDA = stabilogram diffusion analysis.

Phobic postural vertigo (PPV), a subtype of functional dizziness, is characterized by dizziness and subjective imbalance during stance and gait despite normal performance in otoneurologic and clinical balance tests. ${ }^{1} \mathrm{PPV}$, a highly prevalent cause of chronic vertigo/dizziness, significantly compromises functioning and quality of life. Symptoms either arise spontaneously or are triggered by specific perceptual stimuli or social situations. Consequently, patients report an increased fear of falling despite having a normal fall prevalence. ${ }^{2}$

The apparent dissociation between subjective imbalance and objectively maintained balance capabilities in PPV is thought to result from an inadequate but nevertheless functioning postural control strategy. ${ }^{1}$ Accordingly, patients exhibit characteristic alterations in postural control, i.e., a constrained mode of standing with high-frequency postural sway and elevated muscular energy consumption. ${ }^{3}$ It was speculated that this inadequate mode of balance regulation possibly originates from an anxiety-driven increase of attention to postural adjustments, which is accompanied by a stiffening of the musculoskeletal system. ${ }^{1,3}$

From the German Center for Vertigo and Balance Disorders (M.W., T.B., R.S.), Institute for Clinical Neurosciences (T.B.), and Department of Neurology (R.S.), University of Munich, Germany.

Go to Neurology.org for full disclosures. Funding information and disclosures deemed relevant by the authors, if any, are provided at the end of the article. 
The aim of this study was to elucidate these hitherto hypothetical causes of inadequate balance regulation in patients with PPV to improve the diagnosis and therapeutic options for this prevalent form of chronic dizziness. We therefore examined the differential effects of attention on balance control in patients and the amount of antigravity muscle cocontraction occurring during their stance performance.

METHODS Standard protocol approvals, registrations, and patient consents. The study protocol has been approved by the Ethics Committee of the University of Munich and was registered (DRKS00010113). All procedures were in accordance with the Helsinki declaration, and patients gave their written informed consent.

Participants. Ten patients with PPV (age $43.5 \pm 6.2$ years, 6 female, no indication for prior vestibular disorders) and 10 healthy controls (HCs; age $40.3 \pm 4.6$ years, 3 female) participated in the study. The diagnosis of PPV was based on the established criteria, ${ }^{1}$ which have been proven highly reliable in a long-term follow-up (5-15 years) in 106 patients. ${ }^{4}$

Procedures. Postural performance of patients and HCs was evaluated under 4 conditions (each 30-second duration): single-task standing with eyes open and eyes closed and dual-task standing with eyes open and eyes closed. The cognitive dual task consisted of naming items from a given category. Cognitive performance during the dual task was compared to that during the cognitive single task while the participant was sitting quietly. The number of items was documented for each test.

Muscle activity was measured via surface EMG (Telemyo 2400; Noraxon, Scottsdale, AZ) at 1,500 Hz. Bipolar surface electrodes were placed on the tibialis anterior (flexor) and the soleus muscle (extensor) of the dominant leg side. Raw EMG signals were band-pass filtered at 20 to $500 \mathrm{~Hz}$. EMG muscle activity was additionally recorded during maximal voluntary contraction of the soleus (during maximal isometric plantar flexion) and tibialis anterior (during maximal isometric dorsiflexion of the ankle at $90^{\circ}$ ). ${ }^{5}$ Body sway was measured on a stabilometer platform (Kistler 9261A; Kistler Group, Winterthur, Switzerland) at $100 \mathrm{~Hz}$. The center-of-pressure trajectories in the anterior-posterior and mediolateral planes were filtered with a second-order low-pass Butterworth filter with a $10-\mathrm{Hz}$ cutoff frequency.

Data analysis. EMG signals were normalized by maximal voluntary contraction activity. The relative level of leg muscle cocontraction was calculated by the cocontraction index according to the approach of Falconer and Winter as described by Nagai et $\mathrm{al}^{5}$ (figure $1 \mathrm{~A}$ ).

Balance control was studied by the root mean square (RMS) of postural sway and stabilogram diffusion analysis $(\mathrm{SDA})^{6}$ that previously revealed specific balance alterations in patients with PPV. ${ }^{3}$ SDA plots the mean squared center-of-pressure displacement $\left(\Delta r^{2}\right)$ as a function of the time interval ( $\Delta \mathrm{t}$, a moving time window). SDA plots exhibit 2 regions (short-term and long-term diffusion) divided

Figure 1 Neuromuscular regulation of balance in PPV

A

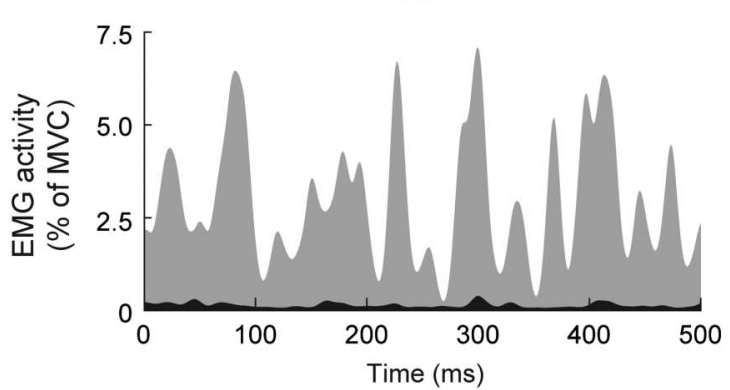

B

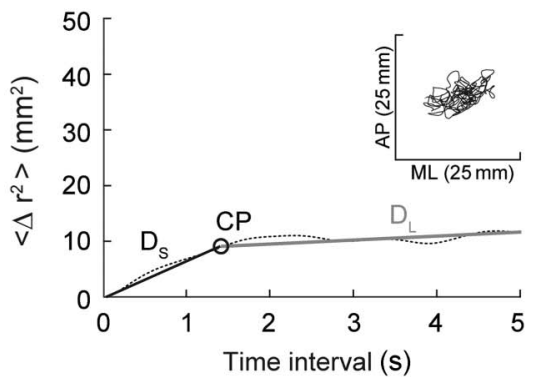

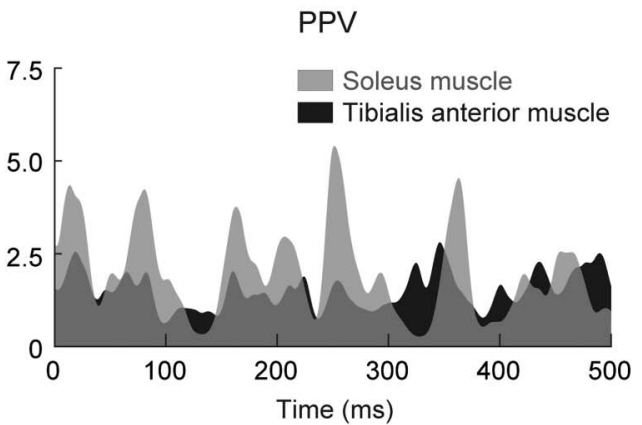

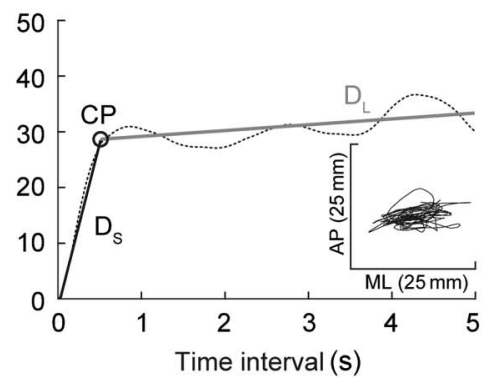

(A) Representative EMG plots of tibialis anterior and soleus muscle activity (rectified, filtered, and normalized with respect to EMG amplitudes during maximal voluntary contraction [MVC]) of one healthy control $(\mathrm{HC})$ and one patient with phobic postural vertigo (PPV) while standing with eyes open; (B) corresponding center-of-pressure trajectories (in anterior-posterior [AP] and mediolateral [ML] plane) and stabilogram diffusion plots of the 2 participants during the same stance trial. Balance control of the patient with PPV is characterized by enhanced cocontraction of leg antigravity muscles (cocontraction index of $51 \%$ vs $5 \%$ in the $\mathrm{HC}$ ). This corresponds to an increased short-term diffusion activity $\left(D_{s}\right)$, indicating abnormal open-loop control, and a shortened critical time interval (CP), implying a precipitate intervening of closed-loop feedback control into the postural control scheme. $\Delta \mathrm{r}^{2}=$ mean squared center-of-pressure displacement. 
by the coordinates of the critical point (critical time $\left[\Delta \mathrm{t}_{\mathrm{c}}\right]$ in seconds and critical displacement $\left[\Delta r^{2}\right]_{c}$ in millimeters squared), which are defined by the intersection of the regression lines fitted to these regions (short- and long-term diffusion coefficients $\left[D_{S}\right.$ and $\left.D_{L}\right]$ in millimeters squared per second). Accordingly, SDA indicates that open-loop feedforward control, which determines the steady-state activity of antigravity muscles, governs balance behavior over shortterm intervals, whereas long-term intervals are regulated by closedloop sensory feedback control that corrects drifts away from the desired posture (figure 1B).

Statistical analysis. Data are reported as mean \pm SE. The effects of each dependent variable were analyzed by repeated-measures analysis of variance and Bonferroni post hoc analysis with group ( $\mathrm{HC} / \mathrm{PPV})$, vision (eyes open/eyes closed), task (single/dual task), and plane (anterior-posterior/mediolateral) as factors. Pearson correlations were performed between cocontraction and SDA outcomes. Results were considered significant at $p<0.05$.

RESULTS During the single task, patients exhibited increased leg muscle cocontractions for standing with eyes open or closed $(p=0.003)$. However, during the dual task, cocontractions in patients normalized to those in HCs. Visual deprivation did not have any effect on cocontractions in patients or HCs. SDA during the single task revealed increased RMS $(p=0.007)$ and short-term diffusion activity $\left(\mathrm{D}_{\mathrm{S}}\right)(p=0.022)$ in patients compared to HCs for standing with eyes open or closed. Increased $D_{S}$ was accompanied by a shortened $\Delta \mathrm{t}_{\mathrm{c}}(p=0.003)$ and increased $\left(\Delta \mathrm{r}^{2}\right)_{\mathrm{c}}$ $(p=0.001)$. However, during the dual task, differences between patients and HCs disappeared. Visual deprivation resulted in higher RMS $(p<$ $0.001), \mathrm{D}_{\mathrm{S}}(p=0.025)$, and $\left(\Delta \mathrm{r}^{2}\right)_{\mathrm{c}}(p<0.001)$. Correlation analysis revealed an association of increased leg muscle cocontractions with higher $D_{S}$ $(R=0.52, p=0.001)$ and shortened $\Delta \mathrm{t}_{\mathrm{c}}(R=$ $0.44 ; p=0.004$ ) (figure 2 and table).

Cognitive performance counts did not differ between HCs and patients or between cognitive single- and dual-task conditions.

Figure 2 Cocontraction of leg antigravity muscles and analysis of body sway behavior
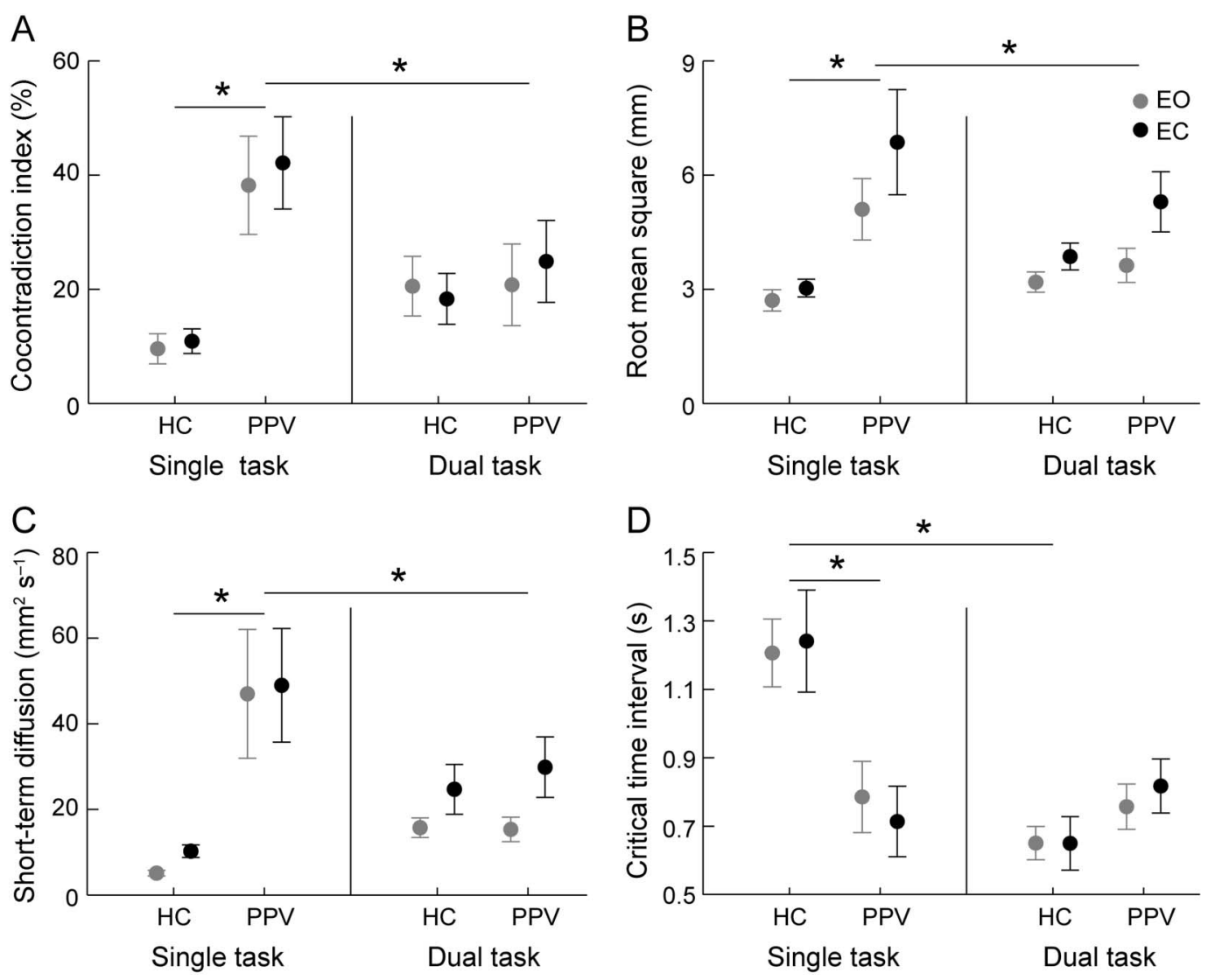

(A) Cocontraction indexes of the examined leg muscle pair (i.e., tibialis anterior and soleus muscle) and (B) root mean square of postural sway, (C) short-term (open-loop) diffusion coefficients, and (D) critical time intervals in healthy controls (HCs) and patients with phobic postural vertigo (PPV) for the 4 studied stance conditions: standing with eyes open (EO) vs standing with eyes closed (EC) (single task) and standing with EO vs standing with EC while performing a cognitive dual task. During a single task, patients exhibited an inadequate neuromuscular regulation of balance, characterized by enhanced cocontractions of antigravity muscles, increased root mean square of postural sway, and short-term diffusion activity (indicating abnormal open-loop control), as well as a shortened critical time interval (implying that the primary sensory feedback threshold of the postural control system is lowered). Balance regulation, however, normalized when patients were distracted by performing a cognitive dual-task. *Significant difference. 


\begin{tabular}{|c|c|c|c|c|c|c|}
\hline \multirow[t]{2}{*}{ Table } & \multicolumn{6}{|c|}{ Descriptive statistics (mean $\pm \mathrm{SE}$ ) and repeated-measures analysis of variance for the EMG and body sway measures } \\
\hline & Leg $\mathrm{Cl}, \%$ & RMS, mm & $\mathrm{D}_{\mathrm{s}}, \mathrm{mm}^{2} / \mathrm{s}$ & $\mathrm{D}_{\mathrm{L}, \mathrm{mm}} / \mathrm{s}$ & $\Delta t_{c}, s$ & $\left(\Delta r^{2}\right)_{c} \mathrm{~mm}^{2}$ \\
\hline \multicolumn{7}{|l|}{ EO ST } \\
\hline HC & $9.6 \pm 2.6$ & $2.7 \pm 0.3$ & $5.1 \pm 0.7$ & $0.8 \pm 0.3$ & $1.2 \pm 0.1$ & $10.9 \pm 1.5$ \\
\hline PPV & $38.2 \pm 8.6$ & $5.1 \pm 0.8$ & $47.0 \pm 15.0$ & $1.1 \pm 0.6$ & $0.8 \pm 0.1$ & $31.6 \pm 5.7$ \\
\hline \multicolumn{7}{|l|}{ EC ST } \\
\hline $\mathrm{HC}$ & $10.9 \pm 2.2$ & $3.0 \pm 0.2$ & $10.2 \pm 1.5$ & $0.2 \pm 0.1$ & $1.2 \pm 0.2$ & $22.1 \pm 3.2$ \\
\hline PPV & $42.2 \pm 8.1$ & $6.9 \pm 1.4$ & $49.0 \pm 13.3$ & $1.3 \pm 1.0$ & $0.7 \pm 0.1$ & $45.0 \pm 6.5$ \\
\hline \multicolumn{7}{|l|}{ EO DT } \\
\hline $\mathrm{HC}$ & $20.6 \pm 5.2$ & $3.2 \pm 0.3$ & $15.7 \pm 2.3$ & $0.6 \pm 0.2$ & $0.7 \pm 0.1$ & $19.5 \pm 3.2$ \\
\hline PPV & $20.8 \pm 7.2$ & $3.6 \pm 0.4$ & $15.3 \pm 2.9$ & $0.9 \pm 0.3$ & $0.8 \pm 0.1$ & $31.9 \pm 11.0$ \\
\hline \multicolumn{7}{|l|}{ EC DT } \\
\hline $\mathrm{HC}$ & $18.3 \pm 4.5$ & $3.9 \pm 0.4$ & $24.7 \pm 5.8$ & $0.8 \pm 0.2$ & $0.7 \pm 0.1$ & $29.0 \pm 5.4$ \\
\hline \multirow[t]{2}{*}{ PPV } & $24.9 \pm 7.2$ & $5.3 \pm 0.8$ & $29.9 \pm 7.1$ & $1.2 \pm 0.5$ & $0.8 \pm 0.1$ & $44.1 \pm 7.5$ \\
\hline & Group (HC|PPV) & Plane (AP|ML) & Vision (EO|EC) & Task (ST|DT) & Group $\times$ vision & Group $\times$ task \\
\hline \multicolumn{7}{|l|}{ EMG } \\
\hline Leg $\mathrm{Cl}$ & $F_{1,18}=4.9, p=0.040^{a}$ & & $F_{1,18}=1.4, p=0.247$ & $F_{1,18}=1.1, p=0.308$ & $F_{1,18}=2.3, p=0.151$ & $F_{1,18}=11.6, p=0.003^{a}$ \\
\hline \multicolumn{7}{|c|}{ Body sway } \\
\hline RMS & $F_{1,36}=7.2, p=0.011^{a}$ & $F_{1,36}=5.4, p=0.026^{a}$ & $F_{1,36}=14.6, p=0.001^{a}$ & $F_{1,36}=7.5, p=0.336$ & $F_{1,36}=14.9, p=0.042^{a}$ & $F_{1,36}=6.0, p=0.019^{a}$ \\
\hline $\mathrm{D}_{\mathrm{s}}$ & $F_{1,36}=6.0, p=0.020^{a}$ & $F_{1,36}=2.2, p=0.147$ & $F_{1,36}=5.5, p=0.025^{a}$ & $F_{1,36}=1.2, p=0.276$ & $F_{1,36}=1.3, p=0.256$ & $F_{1,36}=4.8, p=0.034^{a}$ \\
\hline$D_{L}$ & $F_{1,36}=3.1, p=0.088$ & $F_{1,36}=4.2, p=0.057$ & $F_{1,36}=0.3, p=0.613$ & $F_{1,36}=0.2, p=0.658$ & $F_{1,36}=0.9, p=0.349$ & $F_{1,36}=1.0, p=0.315$ \\
\hline$\Delta t_{c}$ & $F_{1,36}=1.0, p=0.329$ & $F_{1,36}=0.8, p=0.382$ & $F_{1,36}=0.1, p=0.733$ & $F_{1,36}=10.0, p=0.003^{a}$ & $F_{1,36}=0.4, p=0.552$ & $F_{1,36}=19.8, p<0.001^{a}$ \\
\hline$\left(\Delta r^{2}\right)_{c}$ & $\mathrm{~F}_{1,36}=12.4, p=0.001^{\mathrm{a}}$ & $F_{1,36}=16.3, p<0.001^{a}$ & $F_{1,36}=24.2, p<0.001^{a}$ & $F_{1,36}=0.1, p=0.883$ & $F_{1,36}=1.3, p=0.259$ & $F_{1,36}=3.7, p=0.062$ \\
\hline
\end{tabular}

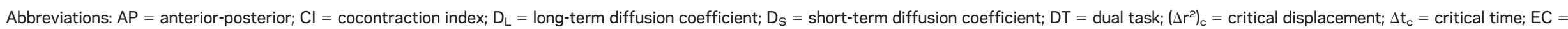
eyes closed; $\mathrm{EO}=$ eyes open; $\mathrm{HC}=$ healthy control; $\mathrm{ML}=$ mediolateral; $\mathrm{PPV}=$ patients with phobic postural vertigo; $\mathrm{RMS}=$ root mean square; $\mathrm{ST}=$ single task.

${ }^{a}$ Significant effects. 
DISCUSSION During quiet standing, patients exhibited increased cocontractions of lower-limb antigravity muscles, causing a stiffening of the musculoskeletal system that is a known phenomenon in anxiety-related disorders such as acrophobia. ${ }^{7}$ This implies that patients apply an inappropriate postural control mode of caution at baseline, which is usually observed only in HCs when they consciously concentrate on balance adjustments (because of a complex balance task ${ }^{3}$ or a postural threat ${ }^{8}$ ) or in the elderly as a compensatory strategy to maintain joint stability. ${ }^{5}$ An active stiffening of the musculoskeletal system constrains the flexibility of postural adjustments (particularly causing a lower net torque around the ankle) and compromises responses to unexpected perturbations.

Increased muscle cocontraction had direct consequences for the sway behavior of patients. Fluctuations within the force output of skeletal muscles increase with muscle activity, which results in enhanced short-term, open-loop body sway as observed in patients. Furthermore, patients exhibited a shortening of the transition interval between open- and closedloop postural regulation that reflects a lowered threshold of postural motor responses to sensory feedback. ${ }^{6}$ This agrees with the assumption that patients with PPV may be afflicted by an oversensitivity to any afferent input that indicates impending destabilization. ${ }^{9}$

Cognitive dual-task interference during standing of HCs results in minor to moderate deterioration of posture, depending on the balance condition. ${ }^{10}$ In contrast, muscle activity and body sway of patients with PPV considerably improved and normalized to that of HCs during the cognitive dual task, i.e., when their focus of attention was distracted from consciously monitoring balance adjustments. This observation is consistent with patients reporting an improvement of their complaints when occupied with sports or other distracting activities. ${ }^{1}$ Such effects of distraction are clinically also well established for other movement disorders not related to anxiety such as psychogenic tremor or dystonia. The converse dualtask effects in PPV imply that balance control in patients at baseline is characterized by an exaggerated attentional involvement. Our data further indicate that patients' preoccupation with conscious monitoring of postural adjustments triggers their inadequate mode of neuromuscular balance regulation. In contrast, withdrawal of visual feedback had only moderate effects on patients' posture, in agreement with earlier findings. ${ }^{3}$
Increased attention on postural control in patients with PPV promotes a vicious circle of symptom emergence by triggering an inadequate neuromuscular balance regulation that results in subjective imbalance, which further enhances anxious control of posture. ${ }^{3}$ This cycle can be broken by distracting patients, which may therefore present an effective coping strategy for preventing or minimizing PPV attacks in susceptible patients.

\section{AUTHOR CONTRIBUTIONS}

M. Wuehr, T. Brandt, and R. Schniepp conceptualized and designed the study. M. Wuehr and R. Schniepp drafted the manuscript. M. Wuehr, T. Brandt, and R. Schniepp analyzed and interpreted the data. All authors read and approved the final manuscript.

\section{STUDY FUNDING}

This work was supported by the German Hertie Foundation and the German Federal Ministry of Education and Research (01EO1401).

\section{DISCLOSURE}

The authors report no disclosures relevant to the manuscript. Go to Neurology.org for full disclosures.

Received June 25, 2016. Accepted in final form October 5, 2016.

\section{REFERENCES}

1. Brandt T. Phobic postural vertigo. Neurology 1996;46: 1515-1519.

2. Schlick C, Schniepp R, Loidl V, Wuehr M, Hesselbarth K, Jahn K. Falls and fear of falling in vertigo and balance disorders: a controlled cross-sectional study. J Vestib Res 2016;25:241-251.

3. Wuehr M, Pradhan C, Novozhilov S, et al. Inadequate interaction between open- and closed-loop postural control in phobic postural vertigo. J Neurol 2013;260:1314-1323.

4. Huppert D, Strupp M, Rettinger N, Hecht J, Brandt T. Phobic postural vertigo: a long-term follow-up (5 to 15 years) of 106 patients. J Neurol 2005;252:564-569.

5. Nagai K, Yamada M, Mori S, et al. Effect of the muscle coactivation during quiet standing on dynamic postural control in older adults. Arch Gerontol Geriatr 2013;56:129-133.

6. Collins JJ, De Luca CJ. Open-loop and closed-loop control of posture: a random-walk analysis of center-ofpressure trajectories. Exp Brain Res 1993;95:308-318.

7. Wuehr M, Kugler G, Schniepp R, et al. Balance control and anti-gravity muscle activity during the experience of fear at heights. Physiol Rep 2014;2:e00232.

8. Holmberg J, Tjernstrom F, Karlberg M, Fransson PA, Magnusson M. Reduced postural differences between phobic postural vertigo patients and healthy subjects during a postural threat. J Neurol 2009;256:1258-1262.

9. Querner V, Krafczyk S, Dieterich M, Brandt T. Phobic postural vertigo: body sway during visually induced roll vection. Exp Brain Res 2002;143:269-275.

10. Melzer I, Benjuya N, Kaplanski J. Age-related changes of postural control: effect of cognitive tasks. Gerontology 2001;47:189-194. 